\title{
The use of a non-biological, bridging, antiprotrusio cage in complex revision hip arthroplasty and periacetabular reconstructive oncologic surgery. Is still today a valid option?: A mid/long-term survival and complications' analysis
}

\author{
Matteo Innocenti ${ }^{1}$ (D) Francesco Muratori ${ }^{1}$. Giacomo Mazzei ${ }^{1}$. Davide Guido ${ }^{1}$. Filippo Frenos ${ }^{1}$. \\ Ersilia Lucenteforte $^{2} \cdot$ Rodolfo Capanna $^{3} \cdot$ Domenico Andrea Campanacci $^{1}$
}

Received: 20 September 2020 / Accepted: 30 April 2021 / Published online: 24 May 2021

(c) The Author(s) 2021

\begin{abstract}
Introduction Burch-Schneider-like antiprotrusio cages (B-SlAC) still remain helpful implants to bridge severe periacetabular bone losses. The purpose of this study was to evaluate outcomes and estimate both cages' failures and complication risks in a series of B-SlAC implanted in revision of failed total hip arthroplasties (THA) or after resection of periacetabular primary or secondary bone malignancies. Risk factors enhancing the chance of dislocations and infections were checked.

Materials and methods We evaluated 73 patients who received a B-SIAC from January 2008 to January 2018. Group A, 40 oncological cases (22 primary tumors; 18 metastases); Group B, 33 failed THAs. We compared both Kaplan-Meier estimates of risk of failure and complication with the cumulative incidence function, taking account the competing risk of death. Cox proportional hazards model was utilized to identify possible predictors of instability and infection. Harris hip score HHS was used to record clinical outcomes.

Results Medium follow-up was 80 months (24-137). Average final HHS was 61 (28-92), with no differences within the two groups $(p>0.05)$. The probabilities of failure and complications were $57 \%$ and $26 \%$, respectively, lower in the oncologic group than in the rTHA group ( $p=0.176$; risk 0.43) $(p=0.52$; risk 0.74$)$. Extended ileo-femoral approach and proximal femur replacement $(p=0.02$, risk ratio $=3.2 ; p=0.04, \mathrm{rr}=2.1$ ) were two significant independent predictors for dislocations, while belonging to group $\mathrm{B}(p=0.04, \mathrm{rr}=2.6)$ was predictable for infections.

Conclusion Burch-Schneider-like antiprotrusio cages are a classical non-biological acetabular reconstruction method that surgeons should bear in mind when facing gross periacetabular bone losses, independently of their cause. However, dislocation and infection rates are high. Whenever possible, we suggest preserving the proximal femur in revision THA, and to use a less-invasive postero-lateral approach to reduce dislocation rates in non-oncologic cases.
\end{abstract}

Keywords Antiprotrusio cages $\cdot$ Revision total hip arthoplasty $\cdot$ Periacetabular bone losses $\cdot$ Pelvic discontinuity $\cdot$ Primary pelvic bone tumors $\cdot$ Periacetabular bone metastases

Matteo Innocenti

innocenti.matteo11@gmail.com

Francesco Muratori

fmuratori@inwind.it

Giacomo Mazzei

drgiacomomazzei@gmail.com

Davide Guido

guidodavide19@gmail.com

Filippo Frenos

frenosfilippo@gmail.com

Ersilia Lucenteforte

ersilia.lucenteforte@unipi.it
Rodolfo Capanna

capanna.rodolfo@gmail.com

Domenico Andrea Campanacci

domenicoandrea.campanacci@unifi.it

1 Department of Orthopaedic Oncology and Reconstructive Surgery, Azienda Ospedaliero-Universitaria Careggi, University of Florence, Largo Palagi 1, 50139 Florence, Italy

2 Unit of Medical Statistics, Department of Clinical and Experimental Medicine, University of Pisa, Pisa, Italy

3 Department of Orthopaedic and Trauma Surgery, Azienda Ospedaliera Universitaria Pisana, Pisa, Italy 


\section{Introduction}

Revisions of total hip arthroplasty (rTHA) and periacetabular resections due to primary or secondary bone malignancies often pose surgeons to face the same problem, a major acetabular bone loss [1-3]. This is usually combined to poor quality of the surrounding pelvic bones becoming a true surgical challenge, particularly when bone loss results in compromise of acetabular column support. Several methods of acetabular reconstruction along with various biologic and non-biologic materials to supplement the periacetabular bone stock have already been described in literature, each one with its strengths and weaknesses [4, 5]. However, what finally drives the surgeon through the choice among different types of reconstruction techniques is the degree of the bone loss itself $[2,6]$. Nowadays the customized triflange 3D printed acetabular components [7] or other custom-made prostheses [8, 9] are becoming more and more popular. However, Burch-Schneider-like antiprotrusio cages, combined or not with massive allograft or morselized grafts and/or cement, still remain a useful and relatively low-cost method in case of severe acetabular/periacetabular bone loss otherwise not bridgeable with simple cementless hemispherical trabecular metal cup [10-13]. Considering the fact that those cages do not rely upon a biological fixation but are simply mechanically fixed to the pelvis, there is certainly a risk for cage breakage and subsequent failure over time. This is even more likely in juvenile high-demand patients who face against periacetabular malignant bone tumors [13]. Furthermore, this is a complex surgery that often requires prolonged surgical time and large surgical exposures in patients with comorbidities related to their age or treated preoperatively with radio- and/or chemotherapy, with all the consequences that it carries in terms of intra- and postoperative complications. Indeed, due to the intra-pelvic extension of the malignancy or its closeness to the mayor iliac vessels as well as the presence of a cup protrusio or a huge intra-pelvic pseudotumor, surgical approaches could range from the classical postero-lateral approach to more invasive ones, such as extended Smith-Pethersen ilio-femoral approach or Enneking's modified ilio-femoral approach. Those approaches are often more invasive for the surrounding soft tissues and they can lead to a higher short-term post-operative instability/dislocation rate irrespective of the type or the position of the prosthetic component used [10, 14].

Therefore, the aim of our study was to evaluate the clinical outcome, and estimate both the risk of cages' failure and complications in a population of patients in which we implanted Burch-Schneider-like antiprotrusio cage due to failure of THA or periacetabular bone loss after resection of primary and secondary bone malignancies. Moreover, we investigated whether the surgical approach as well as the presence of a proximal femur replacement or the reason for implanting the antiprotrusio cage (revision THA versus oncologic surgery) could result a risk factor enhancing the chance of dislocation and/or infection and therefore predict the outcome of such complex patients.

\section{Patients and methods}

\section{Patient selection}

We retrospectively evaluated a cohort of 73 patients who have received a Burch-Schneider-like antiprotrusio cage from January 2008 to January 2018 due to periacetabular bone loss. Forty-five were females and 28 males with an average age of 64 years old (14-93) and average BMI (body mass index) of 24.9 (19-32). Patients were divided into two groups (group A and group B) based on the cause leading to the periacetabular bone defects.

Group A was made up of 40 oncological cases of which 22 had a primary pelvic bone tumor and 18 an acetabular/ periacetabular bone metastases. Enneking e Dunham classification was used to describe the entity of bone defect related to the oncological resection [15]. Seventeen cases underwent a resection type II, 22 type I-II-III resections and one type II-III resection (Table 1).

Group B consisted of 33 patients with a periacetabular bone defect due to failure of THA. The causes of failure were aseptic loosening and THA infection in 24 and 9 cases, respectively. Ten of those patients had a history of hip dysplasia and they had already undergone multiple hip surgeries. In those non-oncological cases, we classified periacetabular bone losses according to Paprosky classification [16]. In eight cases, we found a pelvic discontinuity, nine were $3 \mathrm{~A}$ type bone loss, five $3 \mathrm{~B}$, seven $2 \mathrm{~B}$ and three cases 2C type bone loss (Table 2).

Inclusion criteria were the following: minimum follow-up of 2 year; Paprosky $\geq 2$ B type bone loss or Enneking type II, type II-III and type I-II-III localization of primary/secondary bone tumors; implantation of an acetabular antiprotrusio cage "Burch-Schneider-like"; second-stage reimplantation in patients with a previous history of periprosthtic infection who already underwent the first-stage treatment with removal of the prosthesis, implantation of an antibioticloaded cement spacer, e.v. prolonged antibiotic treatment and who had been tested and excluded (blood test: CRP, ESR; joint aspiration and culture) for any presence of active/ recurrent infection at the time of the index surgery.

We excluded patients who underwent an implantation of any other devices to by-pass the acetabular/periacetabular bone loss rather than the "Burch-Schneider-like" 
Table 1 Demographic characteristics of Group A: periacetabular bone defects due to primary pelvic bone tumor or bone metastases

\begin{tabular}{|c|c|c|c|c|c|c|c|c|c|}
\hline $\begin{array}{l}\text { Patient } \\
\text { number }\end{array}$ & Age (years) & Histotype & $\begin{array}{l}\text { Enneking e } \\
\text { Dunham }\end{array}$ & $\begin{array}{l}\text { Follow-up } \\
\text { (months) }\end{array}$ & $\begin{array}{l}\text { RF survival } \\
\text { (months) }\end{array}$ & Cotile & PFR & Surgical approach & Graft used \\
\hline 1 & 60 & Renal cancer mtx & Type $1-2-3$ & 86 & 86 & DM & $\mathrm{N}$ & Extended ileo-femoral & Massive allograft \\
\hline 2 & 64 & Renal cancer mtx & Type 2 & 35 & 35 & DM & $\mathrm{N}$ & Extended postero-lateral & None \\
\hline 3 & 59 & GII chondrosarcoma & Type $1-2-3$ & 79 & 79 & TRC & $\mathrm{N}$ & Extended ileo-femoral & Massive allograft \\
\hline 4 & 33 & Leiomyosarcoma & Type $2-3$ & 41 & 41 & TRC & $\mathrm{N}$ & Extended ileo-femoral & None \\
\hline 5 & 33 & GII chondrosarcoma & Type $1-2-3$ & 113 & 113 & TRC & $\mathrm{N}$ & Extended ileo-femoral & Massive allograft \\
\hline 6 & 16 & Ewing sarcoma & Type $1-2-3$ & 37 & 37 & TRC & $\mathrm{N}$ & Extended ileo-femoral & Massive allograft \\
\hline 7 & 48 & Thyroid cancer mtx & Type $1-2-3$ & 36 & 36 & TRC & $\mathrm{Y}$ & Extended ileo-femoral & Massive allograft \\
\hline 8 & 67 & GII chondrosarcoma & Type $1-2-3$ & 74 & 74 & $\mathrm{DM}$ & $\mathrm{N}$ & Extended ileo-femoral & Massive allograft \\
\hline 9 & 66 & Hemangiothelioma & Type $1-2-3$ & 72 & 72 & TRC & $\mathrm{N}$ & Extended ileo-femoral & Massive allograft \\
\hline 10 & 67 & GII chondrosarcoma & Type $1-2-3$ & 30 & 30 & $\mathrm{TRC}$ & $\mathrm{N}$ & Extended ileo-femoral & Massive allograft \\
\hline 11 & 66 & GI chondrosarcoma & Type $1-2-3$ & 83 & 83 & TRC & $\mathrm{N}$ & Extended ileo-femoral & Massive allograft \\
\hline 12 & 51 & Breast cancer mtx & Type 2 & 36 & 36 & DM & $\mathrm{N}$ & Extended postero-lateral & None \\
\hline 13 & 60 & $\begin{array}{l}\text { Dedifferentiated chon- } \\
\text { drosarcoma }\end{array}$ & Type $1-2-3$ & 90 & 1 & DM & $\mathrm{Y}$ & Extended ileo-femoral & Massive allograft \\
\hline 14 & 61 & $\begin{array}{l}\text { Chondrosarcoma } \\
\text { grade.II }\end{array}$ & Type $1-2-3$ & 86 & 86 & TRC & $\mathrm{N}$ & Extended ileo-femoral & Massive allograft \\
\hline 15 & 55 & $\begin{array}{l}\text { Dedifferentiated chon- } \\
\text { drosarcoma }\end{array}$ & Type $1-2-3$ & 33 & 33 & TRC & $\mathrm{N}$ & Extended ileo-femoral & Massive allograft \\
\hline 16 & 45 & Giant cell tumor & Type $1-2-3$ & 132 & 132 & DM & $\mathrm{N}$ & Extended ileo-femoral & Massive allograft \\
\hline 17 & 67 & Leiomyosarcoma & Type $1-2-3$ & 36 & 36 & TRC & $\mathrm{N}$ & Extended ileo-femoral & Massive allograft \\
\hline 18 & 71 & Breast cancer mtx & Type 2 & 75 & 75 & DM & $\mathrm{N}$ & Extended postero-lateral & None \\
\hline 19 & 65 & GII chondrosarcoma & Type $1-2-3$ & 47 & 47 & TRC & $\mathrm{N}$ & Extended ileo-femoral & Massive allograft \\
\hline 20 & 62 & GII chondrosarcoma & Type $1-2-3$ & 41 & 41 & TRC & $\mathrm{N}$ & Extended ileo-femoral & Massive allograft \\
\hline 21 & 30 & $\begin{array}{l}\text { Malignant peripheral } \\
\text { nerve sheath tumor }\end{array}$ & Type $1-2-3$ & 36 & 36 & TRC & $\mathrm{N}$ & Extended ileo-femoral & Massive allograft \\
\hline 22 & 14 & Ewing sarcoma & Type $1-2-3$ & 118 & 118 & TRC & $\mathrm{N}$ & Extended ileo-femoral & Massive allograft \\
\hline 23 & 38 & GII chondrosarcoma & Type $1-2-3$ & 56 & 56 & DM & $\mathrm{N}$ & Extended ileo-femoral & Massive allograft \\
\hline 24 & 53 & Multiple myeloma & Type 2 & 36 & 36 & DM & $\mathrm{N}$ & Extended postero-lateral & None \\
\hline 25 & 67 & Osteoblastoma & Type $1-2-3$ & 46 & 46 & $\mathrm{TRC}$ & $\mathrm{N}$ & Extended ileo-femoral & Massive allograft \\
\hline 26 & 46 & Breast cancer mtx & Type $1-2-3$ & 30 & 30 & TRC & $\mathrm{N}$ & Extended ileo-femoral & Massive allograft \\
\hline 27 & 65 & Breast cancer $\mathrm{mtx}$ & Type 2 & 38 & 38 & DM & $\mathrm{N}$ & Extended postero-lateral & None \\
\hline 28 & 74 & Renal cancer mtx & Type $1-2-3$ & 49 & 49 & DM & $\mathrm{N}$ & Extended ileo-femoral & Massive allogrft \\
\hline 29 & 81 & $\begin{array}{l}\text { Carcinoma of } \\
\text { unknown primary } \\
\mathrm{mtx}\end{array}$ & Type 2 & 36 & 36 & DM & $\mathrm{N}$ & Extended postero-lateral & None \\
\hline 30 & 83 & Multiple myeloma & Type 2 & 38 & 38 & DM & $\mathrm{N}$ & Extended ileo-femoral & None \\
\hline 31 & 85 & Prostate cancer $\mathrm{mtx}$ & Type 2 & 36 & 36 & DM & $\mathrm{N}$ & Extended ileo-femoral & None \\
\hline 32 & 66 & Breast cancer mtx & Type 2 & 36 & 36 & DM & $\mathrm{N}$ & Extended ileo-femoral & None \\
\hline 33 & 42 & Melanoma mtx & Type 2 & 36 & 1 & DM & $\mathrm{N}$ & Extended postero-lateral & None \\
\hline 34 & 75 & Breast cancer $\mathrm{mtx}$ & Type 2 & 36 & 36 & DM & $\mathrm{N}$ & Extended ileo-femoral & None \\
\hline 35 & 71 & Renal cancer mtx & Type 2 & 36 & 36 & DM & $\mathrm{N}$ & Extended ileo-femoral & None \\
\hline 36 & 67 & Breast cancer mtx & Type 2 & 44 & 15 & TRC & $\mathrm{Y}$ & Extended postero-lateral & None \\
\hline 37 & 59 & Breast cancer $\mathrm{mtx}$ & Type 2 & 36 & 36 & DM & $\mathrm{N}$ & Extended ileo-femoral & None \\
\hline 38 & 59 & Multiple myeloma & Type 2 & 39 & 10 & DM & $\mathrm{N}$ & Extended ileo-femoral & CABG \\
\hline 39 & 66 & GII chondrosarcoma & Type 2 & 39 & 39 & DM & $\mathrm{N}$ & Extended ileo-femoral & None \\
\hline 40 & 76 & $\begin{array}{l}\text { Epithelioid angiosar- } \\
\text { coma }\end{array}$ & Type 2 & 40 & 40 & DM & $\mathrm{Y}$ & Extended postero-lateral & None \\
\hline
\end{tabular}

$D M$ dual mobility, $T R C$ total retention cup, $C A B G$ corticocancellous allogenic bone graft, $P F R$ proximal femur replacement, $m t x$ metastasis, $R F$ survival revision free survival (considering any surgery in which at least one component of the implanted prostheses was exchanged)

antiprotrusio cage (Muller-type rings, Ganz-type cages, trabecular-metal cup-cage reconstructions, jumbo cups, customized triflange acetabular components, etc.) and patients with a follow-up less than 24 months. Other exclusion criteria were the following: patients with an active periprosthetic infection or patients who resulted infected at the intraoperative cultures at the time of a second-stage reimplantation due to a previous periprosthetic infection; failures 
of TKA with a periacetabular bone loss less than type 2B according to Paprosky.

\section{Surgical approach and implant type}

The decision-making on surgical approach was based on many factors including intra-pelvic extension of the bone tumor, the closeness of the malignancy to the iliac vessels, the presence of a huge intra-pelvic pseudotumor THA related as well as a pelvic discontinuity associated to an intra-pelvic cup protrusio. In revision THA, whenever possible, we performed the same surgical approach utilized during the first/ previous surgeries. Thus, in 45 patients, we used an extended ileo-femoral approach (four extended Smith-Pethersen iliofemoral approaches and 41 Enneking's modified ilio-femoral approaches) while the remaining 28 patients underwent an extended postero-lateral approach.

For every patient, the bone loss was by-passed through the use of an acetabular antiprotrusio cage: 64 partial pelvic replacement (PPR) cages (Waldemar Link GmbH, Hamburg, Germany) and nine Burch-Schneider reinforcement cages (Zimmer Biomet, Warsaw, IN, USA). The associated cemented acetabular cup component within the cage was a self-retaining liner in 27 cases [13 jump system (Permedica S.p.a., Merate, LC, Italy); 9 UHMWPE cemented acetabular retention cup (Groupe Lépine, Genay, France), 3 lubinus polyethylene acetabular cup (Waldemar Link GmbH, Hamburg, Germany), 2 freedom constrained acetabular liners (Zimmer Biomet, Warsaw, IN, USA)] and a double mobility cup in 46 patients [33 Active Articulation Avantage (Zimmer Biomet, Warsaw, IN, USA), 13 BiMobile (Waldemar Link GmbH, Hamburg, Germany)].

Moreover, in addition to the antiprotrusio cage, to fulfill the associated bone loss, we utilized only cement in 38 cases while in other 35 cases, an allogenic bone bank graft was chosen (corticocancellous allogenic bone graft was used in 13 patients, while in 22 cases with a type I-II-III bone tumor extension and in two cases of pelvic discontinuity associated to cup protrusio, we utilized a massive hemipelvic allograft stabilized with screws or plates and screws to the pubic symphysis and the sacroiliac joint). To enhance primary stability, we added a reinforcement mesh in between the neo-acetabulum and the proximal femur in five cases of which three Trevira tube polyethylene terephthalate (Implantcast Gmbh, Buxtehude, Germany) and two ligament advanced reinforcement system-LARS (surgical implants and devices, Arc-sur-Tille, France). Furthermore, four cases of group A and 13 cases of group B underwent a contemporary proximal femoral replacement Megasystem$\mathrm{C}^{\circledR}$ (Waldemar Link GmbH, Hamburg, Germany).

\section{Clinical and radiological evaluations}

All patients were evaluated pre-operatively by standard radiology (AP and axial hip view) and computerized tomography (CT) scan. Moreover, for patients with bone tumors, a magnetic resonance imaging (MRI) was always performed to evaluate the local extension of the disease and consequently plan the bone resection. Regarding the revision arthroplasty group, independently from the presence of a previous history of periprosthetic infection, every patient was screened both through blood examinations (C-reactive protein-CRP, erythrocyte sedimentation rate-ESR, white blood cellWBC) and by a joint aspiration (for both bacterial culture and assessment of synovial with blood cell count, synovial neutrophil percentage and leukocyte esterase) to rule out any septic condition.

Patients of the revision arthroplasty group were both clinically and radiologically followed up at 30 days, 3 months, 6 months and then yearly, while oncological patients were followed up based on their histological diagnosis. Pre-operatively and then at every clinical evaluation, the modified Harris hip score [17] and the presence of a Trendelenburg gait ware determined for both group of patients. Moreover, during the same review, patients were assessed for radiographic complications (including loosening/cage migration, screw breakage, plate fracture) [18], but only those ones leading a subsequent reoperation were recorded.

\section{Statistical analyses}

Statistical analysis was performed using SPSS ${ }^{\circledR}$ statistics software (IBM ${ }^{\circledR}$, Armonk, New York, USA) and R Studio statistical software V1.3.159 (R-Studio PBC, Boston, Massachusetts, USA). Demographical data such as age and BMI were tested for the normal distribution using the Kolmogorov-Smirnov (K-S) test. The Student t-test was used to compare pre- and post-operative HSS results, while the chi-square test was used to compare pre- and post-operative presence of Trendelemburg gait, both taking a $p$ values $<0.05$ to be statistically significant. In addition, the correlation between Trendelemburg gait and dislocation was performed using the chi-square test taking $p<0.05$ as statistically significant. In case of the $>20 \%$ of cells in a $2 \times 2$ table have expected to count less than 5, a 2-sided Fisher exact test was performed. To take account of the competing risk of death we calculated the cumulative incidence function (CIF) [19], by using the method proposed by Gray [20]. To estimate both the risk of failure and complication among subjects receiving surgery for oncologic reason compared to those receiving surgery for rTHA, we used the proportional subdistribution hazards regression model described in Fine 
Table 2 Demographic characteristics of Group B: periacetabular bone defects due to failure of THA

\begin{tabular}{|c|c|c|c|c|c|c|c|c|c|}
\hline $\begin{array}{l}\text { Patient } \\
\text { number }\end{array}$ & Age (years) & Cause & Paprosky & $\begin{array}{l}\text { Follow- } \\
\text { up } \\
\text { (months) }\end{array}$ & $\begin{array}{l}\text { RF } \\
\text { survival } \\
\text { (months) }\end{array}$ & Cotile & PFR & Surgical Approach & Graft used \\
\hline 1 & 72 & Aseptic loosening & $3 a$ & 22 & 22 & DM & $\mathrm{Y}$ & Extended ileo-femoral & C.A.B.G \\
\hline 2 & 62 & Cup protrusio & $\mathrm{Pd}$ & 50 & 38 & DM & $\mathrm{N}$ & Extended ileo-femoral & C.A.B.G \\
\hline 3 & 76 & Aseptic loosening & $2 b$ & 87 & 87 & DM & $\mathrm{N}$ & Extended postero-lateral & None \\
\hline 4 & 43 & Endopelvic pseudotumor & $\mathrm{Pd}$ & 132 & 6 & $\mathrm{DM}$ & $\mathrm{N}$ & Extended ileo-femoral & C.A.B.G \\
\hline 5 & 80 & Aseptic loosening & $\mathrm{Pd}$ & 41 & 41 & TRC & $\mathrm{N}$ & Extended ileo-femoral & C.A.B.G \\
\hline 6 & 76 & Aseptic loosening & $\mathrm{Pd}$ & 44 & 44 & TRC & $\mathrm{Y}$ & Extended ileo-femoral & C.A.B.G \\
\hline 7 & 72 & Aseptic loosening & $\mathrm{Pd}$ & 82 & 82 & $\mathrm{DM}$ & $\mathrm{Y}$ & Extended ileo-femoral & Massive allograft \\
\hline 8 & 79 & Aseptic loosening & $2 \mathrm{c}$ & 46 & 46 & TRC & $\mathrm{N}$ & Extended postero-lateral & None \\
\hline 9 & 45 & Aseptic loosening & $\mathrm{Pd}$ & 108 & 108 & $\mathrm{DM}$ & $\mathrm{N}$ & Extended postero-lateral & None \\
\hline 10 & 84 & Aseptic loosening & $3 b$ & 98 & 98 & $\mathrm{DM}$ & $\mathrm{N}$ & Extended postero-lateral & C.A.B.G \\
\hline 11 & 91 & Infection & $3 a$ & 52 & 52 & TRC & $\mathrm{N}$ & Extended postero-lateral & None \\
\hline 12 & 63 & Aseptic loosening & $2 b$ & 74 & 74 & $\mathrm{DM}$ & $\mathrm{N}$ & Extended postero-lateral & C.A.B.G \\
\hline 13 & 48 & Aseptic loosening & $2 b$ & 92 & 92 & $\mathrm{DM}$ & $\mathrm{N}$ & Extended postero-lateral & None \\
\hline 14 & 79 & Infection & $3 b$ & 86 & 86 & TRC & $\mathrm{Y}$ & Extended postero-lateral & None \\
\hline 15 & 78 & Aseptic loosening & $2 b$ & 105 & 35 & $\mathrm{DM}$ & $\mathrm{N}$ & Extended postero-lateral & C.A.B.G \\
\hline 16 & 69 & Infection & $3 a$ & 97 & 87 & $\mathrm{DM}$ & $\mathrm{Y}$ & Extended postero-lateral & None \\
\hline 17 & 76 & Aseptic loosening & $2 \mathrm{c}$ & 116 & 24 & TRC & $\mathrm{N}$ & Extended postero-lateral & None \\
\hline 18 & 83 & Infection & $3 a$ & 36 & 36 & $\mathrm{DM}$ & $\mathrm{Y}$ & Extended ileo-femoral & None \\
\hline 19 & 63 & Infection & $3 a$ & 137 & 137 & $\mathrm{DM}$ & $\mathrm{Y}$ & Extended postero-lateral & None \\
\hline 20 & 83 & Infection & $3 a$ & 79 & 79 & TRC & $\mathrm{Y}$ & Extended postero-lateral & None \\
\hline 21 & 63 & Aseptic loosening & $3 a$ & 94 & 94 & $\mathrm{DM}$ & $\mathrm{N}$ & Extended ileo-femoral & None \\
\hline 22 & 59 & Infection & $3 b$ & 132 & 25 & TRC & $\mathrm{Y}$ & Extended ileo-femoral & C.A.B.G \\
\hline 23 & 76 & Cup protrusio & $\mathrm{Pd}$ & 76 & 76 & $\mathrm{DM}$ & $\mathrm{Y}$ & Extended ileo-femoral & None \\
\hline 24 & 57 & Aseptic loosening & $\mathrm{Pd}$ & 78 & 67 & $\mathrm{DM}$ & $\mathrm{N}$ & Extended ileo-femoral & C.A.B.G \\
\hline 25 & 93 & Aseptic loosening & $\mathrm{Pd}$ & 41 & 1 & TRC & $\mathrm{N}$ & Extended postero-lateral & None \\
\hline 26 & 60 & Infection & $3 b$ & 132 & 132 & $\mathrm{DM}$ & $\mathrm{N}$ & Extended postero-lateral & None \\
\hline 27 & 78 & Aseptic loosening & $2 \mathrm{~b}$ & 94 & 94 & $\mathrm{DM}$ & $\mathrm{Y}$ & Extended postero-lateral & C.A.B.G \\
\hline 28 & 67 & Infection & $3 a$ & 57 & 57 & $\mathrm{DM}$ & $\mathrm{N}$ & Extended ileo-femoral & None \\
\hline 29 & 72 & Aseptic loosening & $2 \mathrm{~b}$ & 36 & 36 & $\mathrm{DM}$ & $\mathrm{N}$ & Extended postero-lateral & None \\
\hline 30 & 76 & Aseptic loosening & $3 a$ & 39 & 39 & $\mathrm{DM}$ & $\mathrm{N}$ & Extended ileo-femoral & C.A.B.G \\
\hline 31 & 61 & Aseptic loosening & $2 \mathrm{~b}$ & 47 & 47 & $\mathrm{DM}$ & $\mathrm{Y}$ & Extended ileo-femoral & None \\
\hline 32 & 62 & Aseptic loosening & $3 b$ & 36 & 36 & $\mathrm{DM}$ & $\mathrm{Y}$ & Extended postero-Lateral & None \\
\hline 33 & 74 & Aseptic loosening & $2 \mathrm{c}$ & 38 & 38 & $\mathrm{DM}$ & $\mathrm{N}$ & Extended postero-Lateral & None \\
\hline
\end{tabular}

$P d$ pelvic discontinuity, THA total hip arthroplasty, DM dual mobility, TRC total retention cup, C.A.B.G. corticocancellous allogenic bone graft, $R F$ survival revision free survival (considering any surgery in which at least one component of the implanted prostheses was exchanged)

and Gray [21]. The Cox proportional-hazards regression model was utilized to identify multivariate risk factors predictive of instability and infection [independent variables included the surgical approach used as well as the presence of a proximal femur replacement and the reason for implanting the antiprotrusio cage (revision THA versus oncology surgery)], with the risk ratio and $95 \%$ confidence interval used to measure the strength of the association [22].

\section{Results}

\section{Clinical evaluation}

The average follow-up of the revision THA group was 86 months (24-137 months), while for the oncologic patient group was 74 months (24-122 months). Twenty-one (28.7\%) patients died. The majority of them belonged to the oncologic group where the deaths were $14 / 40$ (35\%) of which nine in patients with secondary bone metastases and five in patients with primary oncological bone disease. The 
histotypes of primary bone tumors in patients who died of disease progression were: spindle cell sarcoma, Ewing sarcoma, dedifferentiated chondrosarcoma, G2 chondrosarcoma and malignant peripheral nerve sheath tumor MPNST (Table 1). Among the revision arthroplasty group, 7 (21\%) patients died due to causes other than the hip revision surgery.

Demographic data, such as age and BMI, were normally distributed (age: $\mathrm{K}-\mathrm{S}=0.153 ; p=0.572$; skewness $=$ - 0.969; kurtosis $=1.136$ ) (BMI: $\mathrm{K}-\mathrm{S}=0.130 ; p=0.060$; skewness $=-0.559$; kurtosis $=-1.06$ ).

The modified Harris hip score showed a significant increase pre- to post-operatively $(p<0.05)$ : an average pre-operative score of 14 (14-32) to an average score of 61 $(28-92)$ at the last follow-up $(p<0.05)$ and it was substantially superimposable within the two groups $(p>0.05)$. We recorded a Trendelemburg gait in 18 patients (24\%) with a significant difference between pre- and post-operative findings $\left(\chi^{2}\right.$ test $\left.=9.56 ; p=0.002\right)$.

We found no association between post-operative Trendelemburg gait and dislocations $\left(\chi^{2}\right.$ test $=0.821$, Fisher exact test $=0.532 ; p=0.365$ ).

\section{Survivorship analysis}

Taking into account the competing risk of death, the estimates of risk of failure at 5 years were 0.1 and 0.19 for the oncologic and the rTHA group, respectively (Table 3 , Fig. 1), while the risk of complication at 5 years was 0.3 and 0.37 for the oncologic and the rTHA group, respectively (Table 3, Fig. 2). Comparing both groups, the probability of failure was 57\% lower in the oncologic group than in the rTHA group without reaching the statistical significance $[p=0.176$; risk 0.43 (CI $0.13-1.42$ )], while the probability of complications was $26 \%$ lower in the oncologic group than in the rTHA group [ $p=0.52$; risk 0.74 (CI 0.37-1.46)] (Table 3).

The Cox proportional hazards regression analyses revealed that the surgical approach used, the presence of a proximal femur replacement and the reason for implanting the cage were significant independent predictor, respectively, for post-operative instability the former two $(p=0.02$, risk ratio $=3.2 ; p=0.04$, risk ratio $=2.1)$ and infections the latter $(p=0.04$, risk ratio $=2.6)$. Specifically, the odds of instability were estimated to be three times higher the 1st year for patients who were treated with an extended ileo-femoral approach and two times higher for those who underwent a complementary proximal femur replacement, as well as the odds of infections were estimated to be more than two times higher for the group in which the reason for implanting a cage was due to revision THA (Table 4).

\section{Complications and failures}

A total of 46 complications were observed in 31 patients (42\%): 24 dislocations (18 primary and six recurrent dislocations), eight peroneal nerve palsies (six transients with full recovery and two persistent; every of those were related to dislocations except one persistent palsy related to the index surgery), ten infections, three aseptic loosening and one local recurrence of the underling oncological disease. Details of complications per patient are reported in Supplementary Material as Tables 5, 6 (the full report of complications is also extensively described in Supplementary Material).

\section{Discussion}

One of the aims of the study was to investigate whether this non-biological type of acetabular reconstruction could keep its mechanical strength with time at mid/long-term followup, independently of the nature of acetabular/periacetabular bone loss. Taking into account the competing risk of death, in our series, the risk of failure was $30 \%$ and $10 \%$ at 10 years
Table 3 Competing risk analysis for failure and complications

\begin{tabular}{lllllllll}
\hline Competing risk analysis for failure & \multicolumn{3}{l}{ Time (months) } & \multicolumn{3}{c}{$p$} & \multirow{2}{*}{$p$ value } \\
\cline { 2 - 7 } & 20 & 40 & 60 & 80 & 100 & 120 & \\
\hline Revision THAs for failure & 0.060 & 0.189 & 0.189 & 0.235 & 0.306 & 0.306 & 0.176 \\
Oncologic resections for failure & 0.100 & 0.100 & 0.100 & 0.100 & 0.100 & 0.100 & \\
Revision THAs for competing death & 0.000 & 0.000 & 0.122 & 0.122 & 0.122 & 0.312 & 0.011 \\
Oncologic resections for competing death & 0.000 & 0.251 & 0.379 & 0.444 & 0.444 & 0.444 & \\
Competing risk analysis for complications & & & & & & & \\
Revision THAs for failure & 0.212 & 0.373 & 0.373 & 0.470 & 0.539 & 0.539 & 0.522 \\
Oncologic resections for failure & 0.275 & 0.300 & 0.300 & 0.300 & 0.300 & 0.300 & \\
Revision THAs for competing death & 0.000 & 0.000 & 0.118 & 0.118 & 0.118 & 0.232 & 0.062 \\
Oncologic resections for competing death & 0.000 & 0.169 & 0.292 & 0.350 & 0.350 & 0.350 & \\
\hline
\end{tabular}




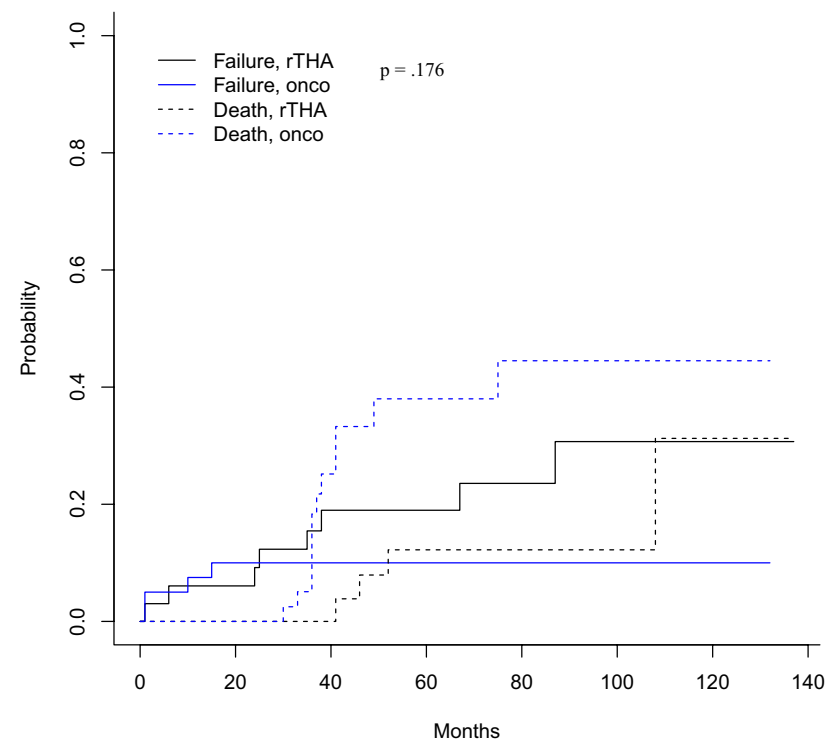

Fig. 1 Cumulative incidence function for cages' failure taking into account of the competing risk of death

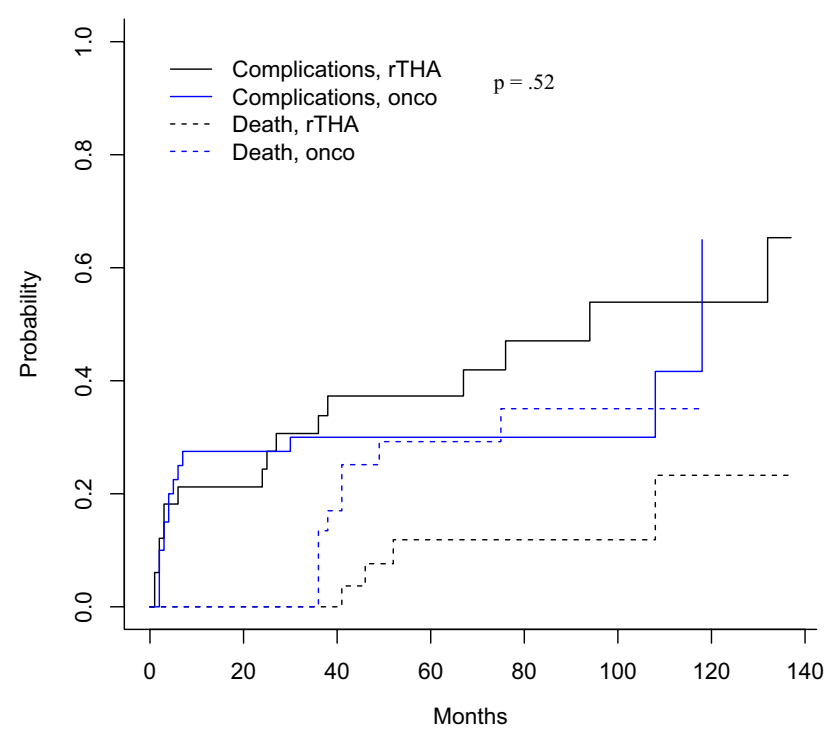

Fig. 2 Cumulative incidence function for complications taking into account of the competing risk of death for the rTHA and oncologic group, respectively, with no differences between the two groups.

The first long-term cages' survival rate was reported by Berry and Müller [23] in which they found 76\% survival rate in 32 hips at 5 years of follow-up. More recently, in the review published by Aprato et al. [24], the survival rate of those particular type of reinforcement rings among 13 different articles ranges from 72 to $100 \%$ at a medium follow-up of 5.6 years.

Rowell et al. [25] were the first to report the treatment of 47 acetabular bone metastasis with the use of PPR link system between 2006 and 2017. They found a surprisingly high survivorship of $91 \%$ free from all-cause revision or reoperation, probably due to the short survival expectancy of metastatic patients.

Focusing on structural/mechanical failure of the cage itself (aseptic loosening/fixation failure/cage breakage), Hsu et al. [14] described a total of six (19.4\%) failures with associated component migration at the latest follow-up (10 years), reporting that three implants had broken iliac screws but no cage breakages occurred. Among our series, we reported only four cases $(5.5 \%)$ of structural/mechanical failure that required a re-intervention equally distributed between the two groups.

Udomkiat et al. [26] retrospectively reviewed a series of 18 Burch-Schneider cages and found a 6-year survivorship for mechanical failure of $63.6 \%$ including both cages revised for aseptic loosening and those that had radiographic loosening but had not been revised.

In line with the previously reported literature $[11,23$, 25, 27-31], we also observed good functional results with a median modified Harris hip score of 61 and no significative differences among the two groups. Nevertheless, we reported a high incidence of Trendelenburg gait (24\%) among both groups, but we found no association in between this particular type of complication and the post-operative dislocation rate $(F=0.532)$. Differently from our report, Udomkiat et al. found that $38.8 \%$ (7 out of 18 patients) of their cages' series had a dislocation. They described that the dislocation rate was related to muscle weakness $(p=0.028)$.
Table 4 Predictor of dislocation and infection after implanting the antiprotrusio cage

\begin{tabular}{|c|c|c|c|c|c|c|}
\hline & \multicolumn{3}{|c|}{ Dislocation } & \multicolumn{3}{|c|}{ Infection } \\
\hline & $p$ value & Odds ratio & $95 \% \mathrm{CI}$ & $p$ value & Odds ratio & $95 \% \mathrm{CI}$ \\
\hline \multicolumn{7}{|l|}{ Surgical approach } \\
\hline Postero-lateral approach & 0.08 & 0.9 & $0.6-1.2$ & 0.48 & 0.76 & $0.6-0.9$ \\
\hline Extended ilio-femoral approach & 0.02 & 3.2 & $2.4-3.6$ & 0.12 & 0.97 & $0.9-1.3$ \\
\hline Proximal femur replacement & 0.04 & 2.1 & $1.9-2.6$ & 0.64 & 0.78 & $0.7-1.0$ \\
\hline \multicolumn{7}{|c|}{ Reason for implanting an antiprotrusio cage } \\
\hline Revision THAs & 0.36 & 0.8 & $0.5-1.3$ & 0.04 & 2.6 & $1.9-3.1$ \\
\hline Oncological resections & 0.09 & 1.2 & $0.7-1.5$ & 0.18 & 1.4 & $1.1-1.6$ \\
\hline
\end{tabular}


Among our study population, we reported a high incidence of complications. Almost half of our patients had at least one complication (42\%). Taking into account the competing risk of death, the risk of getting complication was 53\% and 30\% at 10 years for the rTHA and oncologic group, respectively, with no differences among the groups. The most common complication was dislocation (primary dislocation in $24.7 \%$ of patients) followed by infection (13.7\% of patients).

In our study, we observed the surgical approach/exposure along with the presence of a complementary proximal femur replacement as the two major independent risk factors for dislocation. Indeed, the odd ratio of dislocation resulted three times higher $(p=0.02$, risk ratio $=3.2)$ in those patients who underwent an extended ileo-femoral approach and two times higher $(p=0.04$, risk ratio $=2.1)$ for those who also had a proximal femur replacement. This can be related on the fact that this surgical approach was most frequently used in oncologic or challenging cases, in the presence of huge endopelvic pseudotumor in contact with iliac vessels or endopelvic cup protrusion associated with pelvic discontinuity, in which a postero-lateral approach could not be the first choice. Moreover, all the patients (100\%) of the rTHA group who got a dislocation underwent also a proximal femur replacement and, actually, every patient of the oncologic group who had a proximal femur replacement (four patients) got a dislocation as well, independently of the surgical approach used. Based on the fact that during the implantation of a proximal femur replacement prostheses both the gluteus muscles and the psoas muscle have to be detached, it also remarks the importance that soft tissues play in the stability of a revision THA. It means that both factors, an extensive invasive surgical approach and the presence of a proximal femur replacement, could justify the reason why we had that high rates of dislocation in both groups (dislocation rate: $35 \%$ in group A and $30.3 \%$ in group B).

A lower dislocation rate was reported in the periacetabular metastatic series of patients by Rowell et al. [25] who described four events in 46 patients (9\%). A slightly higher rate of dislocation (17\%) was described by Mark Clayer [12] in a series of 29 patients undergoing implantation of an anti-protrusio cage for metastatic pelvic disease. Such low dislocation rates could be related to the short survival of metastatic patients. Indeed, in the latter report, the median length of patient survival was only 12 months ( 3 days -100 months) after the procedure.

Regarding the second-most common complication, we found that implanting an anti-protrusio-cage due to failure of a previous THA or revision THA was an independent risk factor in predicting post-operative infections. Indeed, the infection rate among the two groups was, respectively, $2.5 \%$ and $24.2 \%$ in the oncologic group and revision THA group and the estimated odds ratio of infections was more than two times higher $(p=0.04$, risk ratio $=2.6)$ for the latter group. Despite no previous study has compared those two different categories of patients, single literature reports are in line with our findings concerning the oncologic group (percentage of infection ranging from 2 to $8 \%)[12,25,27]$ but the infection rates among the revision THA are reported to be lower than what we have found (percentage of infection ranging from 3 to $10 \%$ ) $[14,30$, $31]$. We believe that higher infection rate in our revision THR series can be related to previous multiple surgeries, with an average of 2.9 procedures before the index operation.

This study has several limitations. We studied two nonhomogeneous groups of patients, but our initial purpose was to evaluate the durability of a non-biologic implant in bypassing a gross acetabular/periacetabular bone defect independently of the cause of the bone loss itself. Furthermore, the two groups have in common a poor host bone healing potential, either because of the scarce quality of bone that sustained many previous surgeries [32] or because the bone lesion (e.g. metastasis) is not expected to heal [33] or because the periacetabular bone resection (e.g. primary bone tumors) has been bypassed with a massive allograft [34] that has no capacity to support the bone growth over an ingrowth material (e.g. trabecular metal).

Second, the sample size of our study is not big enough to give a proper consistency at the statistical analysis performed, therefore the interpretation of data cannot be taken as milestone.

Finally, despite we performed X-rays at every out-patient control, we have only recorded those cases that required a subsequent revision surgery due to cage loosening/migration/structural breakage. This could therefore lead to an underestimation of intrinsic cages' mechanical failures.

As far as we know, there are no previously reported studied in literature comparing those two groups of patients with the purpose to evaluate the potentialities and weaknesses of this particular bridging acetabular cage implant, independently of the nature causing the acetabular/periacebular bone defect as well as to investigate whether or not there could exist some difference in complication rates among the two different groups and if those could be related to some specific risk factors.

\section{Conclusion}

Burch-Schneider-like antiprotrusio cages are useful implants when facing against different types of gross acetabular/periacetabular bone losses, with an overall high long-term survival rate. However, complication rate is high, mostly dislocations and infections. Whenever possible, we suggest both to spare the proximal femur along with its muscular insertions in rTHA, and to use a lessinvasive postero-lateral approach to reduce dislocation rates. 
Supplementary Information The online version contains supplementary material available at https://doi.org/10.1007/s00402-021-03929-6.

Acknowledgements We truly appreciated the technical and professional support provided by Prof. Gianni Virgili. He has been like a trigger in making the right choices during the manuscript's revision process.

Authors' contributions MI made both the conception and the design of the work as well as they drafted the first version of the work. Each author has made substantial contributions to the design of the work as well as to the acquisition, analysis and interpretation of data. All authors have also substantively revised the work and has approved the submitted version (and any substantially modified version that involves the author's contribution to the study), and have agreed both to be personally accountable for the author's own contributions and to ensure that questions related to the accuracy or integrity of any part of the work, even ones in which the author was not personally involved, are appropriately investigated, resolved, and the resolution documented in the literature.

Funding Open access funding provided by Università degli Studi di Firenze within the CRUI-CARE Agreement. The authors received no financial support for the research, authorship, and/or publication of this article.

\section{Declarations}

Conflict of interest The authors declare that they have no competing interests. The authors have no conflicts of interest.

Ethical approval All patients accepted the proposed treatment and follow-up after an adequate information and written consent. The study and follow-up, respecting the criteria of the Declaration of Helsinki, have been approved by Institutional Review Board of Azienda Ospedaliera Universitaria Careggi (AOUC) Department of Surgery and Translational Medicine. The Institutional Review Board accepted the proposal of the study, and all selected patients were properly informed before surgery about the treatment and follow-up visits after discharge.

Open Access This article is licensed under a Creative Commons Attribution 4.0 International License, which permits use, sharing, adaptation, distribution and reproduction in any medium or format, as long as you give appropriate credit to the original author(s) and the source, provide a link to the Creative Commons licence, and indicate if changes were made. The images or other third party material in this article are included in the article's Creative Commons licence, unless indicated otherwise in a credit line to the material. If material is not included in the article's Creative Commons licence and your intended use is not permitted by statutory regulation or exceeds the permitted use, you will need to obtain permission directly from the copyright holder. To view a copy of this licence, visit http://creativecommons.org/licenses/by/4.0/.

\section{References}

1. Brown TS, Salib CG, Rose PS et al (2018) Reconstruction of the hip after resection of periacetabular oncological lesions: a systematic review. Bone Jt J 100-B:22-30
2. Sheth NP, Nelson CL, Springer BD et al (2013) Acetabular bone loss in revision total hip arthroplasty: evaluation and management. J Am Acad Orthop Surg 21:128-139

3. Beadel GP, McLaughlin CE, Wunder JS et al (2005) Outcome in two groups of patients with allograft-prosthetic reconstruction of pelvic tumor defects. Clin Orthop Relat Res. https://doi.org/10. 1097/01.blo.0000180048.43208.2f

4. Fryhofer G, Ramesh S, Sheth N (2019) Acetabular reconstruction in revision total hip arthroplasty. J Clin Orthop Trauma 11. https:// doi.org/10.1016/j.jcot.2019.11.004

5. Gamradt SC, Lieberman JR (2003) Bone graft for revision hip arthroplasty: biology and future applications. Clin Orthop Relat Res 417:183-194

6. Shon W, Santhanam S, Choi J (2016) Acetabular reconstruction in total hip arthroplasty. Hip Pelvis 28:1. https://doi.org/10.5371/ hp.2016.28.1.1

7. De Martino I, Strigelli V, Cacciola G et al (2019) Survivorship and clinical outcomes of custom triflange acetabular components in revision total hip arthroplasty: a systematic review. J Arthroplasty 34:2511-2518

8. Müller PE, Dürr HR, Wegener B et al (2002) Internal hemipelvectomy and reconstruction with a megaprosthesis. Int Orthop. https://doi.org/10.1007/s00264-001-0322-4

9. Rudert M, Holzapfel BM, Pilge H et al (2012) Partial pelvic resection (internal hemipelvectomy) and endoprosthetic replacement in periacetabular tumors. Oper Orthop Traumatol. https://doi.org/10. 1007/s00064-012-0161-z

10. Pieringer H, Auersperg V, Böhler N (2006) Reconstruction of severe acetabular bone-deficiency. The Burch-Schneider antiprotrusio cage in primary and revision total hip arthroplasty. $\mathrm{J}$ Arthroplasty. https://doi.org/10.1016/j.arth.2005.02.016

11. Regis D, Sandri A, Bonetti I (2014) Acetabular reconstruction with the Burch-Schneider antiprotrusio cage and bulk allografts: minimum 10-year follow-up results. Biomed Res Int. https://doi. org/10.1155/2014/194076

12. Clayer M (2010) The survivorship of protrusio cages for metastatic disease involving the acetabulum. Clin Orthop Relat Res 468:2980-2984

13. Li D, Guo W, Yang R et al (2011) Utilization of reinforced acetabular cages with caudal flange in reconstructing pelvic defect after acetabular tumor resection. Zhongguo Xiu Fu Chong Jian Wai Ke Za Zhi 25:1409-1413

14. Hsu CC, Hsu CH, Yen SH, Wang JW (2015) Use of the BurchSchneider cage and structural allografts in complex acetabular deficiency: 3- to 10-year follow up. Kaohsiung J Med Sci. https:// doi.org/10.1016/j.kjms.2015.08.001

15. Enneking WF, Dunham WK (1978) Resection and reconstruction for primary neoplasms involving the innominate bone. J Bone Jt Surg Ser A. https://doi.org/10.2106/00004623-197860060-00002

16. Paprosky WG, Perona PG, Lawrence JM (1994) Acetabular defect classification and surgical reconstruction in revision arthroplasty. A 6-year follow-up evaluation. J Arthroplasty. https://doi.org/10. 1016/0883-5403(94)90135-X

17. Kumar P, Sen R, Aggarwal S et al (2019) Reliability of modified Harris hip score as a tool for outcome evaluation of total hip replacements in Indian population. J Clin Orthop Trauma. https:// doi.org/10.1016/j.jcot.2017.11.019

18. Johnston RC, Fitzgerald RH, Harris WH et al (1990) Clinical and radiographic evaluation of total hip replacement. A standard system of terminology for reporting results. J Bone Jt Surg Ser A. https://doi.org/10.2106/00004623-199072020-00002

19. Gillam MH, Ryan P, Graves SE et al (2010) Competing risks survival analysis applied to data from the Australian orthopaedic association national joint replacement registry. Acta Orthop. https://doi.org/10.3109/17453674.2010.524594 
20. Robert JG (1988) A class of K-sample tests for comparing the cumulative incidence of a competing risk. Ann Stat 16(3):1141-1154

21. Fine JP, Gray RJ (1999) A proportional hazards model for the subdistribution of a competing risk. J Amn Stat Assoc 94(446):496509. https://doi.org/10.1080/01621459.1999.10474144

22. Cox DR (1972) Regression models and life-tables. J R Stat Soc B. https://doi.org/10.1111/j.2517-6161.1972.tb00899.x

23. Berry DJ, Muller ME (1992) Revision arthroplasty using an antiprotrusio cage for massive acetabular bone deficiency. J Bone Jt Surg Ser B. https://doi.org/10.1302/0301-620x.74b5.1527119

24. Aprato A, Olivero M, Vergano LB, Massè A (2019) Outcome of cages in revision arthroplasty of the acetabulum: a systematic review. Acta Biomed 90:24

25. Rowell P, Lowe M, Sommerville S, Dickinson I (2019) Is an acetabular cage and cement fixation sufficiently durable for the treatment of destructive acetabular metastases? Clin Orthop Relat Res. https://doi.org/10.1097/CORR.0000000000000725

26. Udomkiat P, Dorr LD, Won YY et al (2001) Technical factors for success with metal ring acetabular reconstruction. J Arthroplasty. https://doi.org/10.1054/arth.2001.27669

27. Hoell S, Dedy N, Gosheger G et al (2012) The Burch-Schneider cage for reconstruction after metastatic destruction of the acetabulum: outcome and complications. Arch Orthop Trauma Surg. https://doi.org/10.1007/s00402-011-1351-0

28. Peters CL, Curtain M, Samuelson KM (1995) Acetabular revision with the Burch-Schnieder antiprotrusio cage and cancellous allograft bone. J Arthroplasty. https://doi.org/10.1016/S08835403(05)80179-2
29. Gill TJ, Sledge JB, Müller ME (1998) The Burch-Schneider antiprotrusio cage in revision total hip arthroplasty. Indications, principles and long-term results. J Bone Jt Surg Ser B. https://doi.org/ 10.1302/0301-620X.80B6.8658

30. Regis D, Sandri A, Bonetti I et al (2012) A minimum of 10-year follow-up of the Burch-Schneider cage and bulk allografts for the revision of pelvic discontinuity. J Arthroplasty. https://doi.org/10. 1016/j.arth.2011.11.019

31. Goodman S, Saastamoinen H, Shasha N, Gross A (2004) Complications of ilioischial reconstruction rings in revision total hip arthroplasty. J Arthroplasty. https://doi.org/10.1016/j.arth.2003. 11.015

32. Gaiani L, Bertelli R, Palmonari M, Vicenzi G (2009) Total hip arthroplasty revision in elderly people with cement and BurchSchneider anti-protrusio cage. Chir Organi Mov. https://doi.org/ 10.1007/s12306-009-0019-1

33. Yazawa Y, Frassica FJ, Chao EYS et al (1990) Metastatic bone disease: a study of the surgical treatment of 166 pathologic humeral and femoral fractures. Clin Orthop Relat Res. https:// doi.org/10.1097/00003086-199002000-00036

34. Campanacci D, Chacon S, Mondanelli N et al (2012) Pelvic massive allograft reconstruction after bone tumour resection. Int Orthop. https://doi.org/10.1007/s00264-012-1677-4

Publisher's Note Springer Nature remains neutral with regard to jurisdictional claims in published maps and institutional affiliations. 